\title{
Empirical Analysis on MSW Optimization Management Technology based on Fuzzy Method
}

\author{
Yanrui Zhang ${ }^{1, *}$ Wenfu $\mathrm{Cao}^{2}$ and Lina Zhang ${ }^{1}$ \\ ${ }^{1}$ College of Sciences, Agricultural University of Hebei, Baoding, 071001, China; ${ }^{2}$ College of Mathematics, Hebei Uni- \\ versity, Baoding, 071002, China
}

\begin{abstract}
The scientific disposal of Municipal Solid Waste (MSW) is of great significance to the urban ecological environment and the residents' health. Taking into account the economic, technical, environmental, social indicators, as well as their subindicators, analyzing fourteen evaluation factors, the paper discusses four kinds of disposal methods of MSW using AHP and Fuzzy comprehensive evaluation. The results indicate that the most suitable waste disposal method is the comprehensive disposal method.
\end{abstract}

Keywords: AHP, fuzzy comprehensive evaluation, index system.

\section{INTRODUCTION}

In recent years, with rapid development of economy and continuous improvements in living standards, the output of municipal solid waste (MSW) is on the increase. Some cities are faced with the issue of being besieged with MSW, which has brought serious harm to the people's health.

The effective methods of solving MSW problem involves volume and weight reduction, harmless treatment such as reutilization and recycle, power-converting. At present, MSW disposal technology involves landfill, compost and incineration. The traditional sanitary landfill requires a lot of land offer, therefore MSW treatment should be diversified and varied according to local environment and economic level $[1,2]$.

Taking Shunyi waste disposal plant located in the suburbs of Beijing (a megalopolis) as research object, this paper establishes comprehensive evaluation index system of MSW disposal. Four kinds of MSW disposal technologies, that is, landfill, compost, incineration, and comprehensive treatment are analyzed by AHP and fuzzy comprehensive evaluation method.

\section{THE ESTABLISHMENT OF INDEX SYSTEM OF MSW DISPOSAL TECHNOLOGY}

A scientific and systematic overall evaluation of index system is the foundation of comprehensive evaluation. This paper selects economic, technological, environmental and social indicators as four first-level indexes, set up several second-level indicators below each first-level indexes, and constructed a comprehensive evaluation index system of MSW disposal method (Table 1) [3-5].

\subsection{Determination of Index Weights by AHP Method}

The judgment matrix is given on the basis of some institutions and experts on MSW disposal technology consulting. The first-level and the second-level weights are calculated by AHP. Table 2 indicates the process of four B layers of index weight calculation. $\omega_{i}$ is the weight coefficient, $\lambda_{\max }$ is the largest eigen value of the judgment matrix, $R I$ is random consistency index. When $C R<0.1$, the judgment matrix has consistent satisfactory values.

Similarly, the second-level weights are obtained as follows: (in Table $\mathbf{3}$ ).

\subsection{The Selection of Membership Function}

Different membership degree functions are given for all second-level indexes. Here the total project investment (a second-level index) is taken as a demonstration. Due to the difference of technological level, the average investment date of three MSW disposal method (sanitary, landfill, compost, incineration) are not precise, so the experience data are adopted. The average investment of landfill is about 600 1500 million /100 tons; the average investment of compost is about 500-4000 million /100 tons; the average investment of incineration technology is about 3500-6500 million /100 tons; the average investment of comprehensive treatment is about 1500-3000 million /100 tons. Due to the large differences of four MSW disposal method investment amounts, different membership degree functions are given corresponding to four MSW disposal methods (Table 4) $[6,7]$. The evaluation set is given, $U_{11}=(H, G, M, L)$ that is, (higher investment amount, high investment amount, middle investment amount, low investment amount). The establishment of the membership function are four kinds of treatment technology on the $U_{11}$. 
Table 1. Index system of MSW disposal technology.

\begin{tabular}{|c|c|c|}
\hline Target Layer & The First Level & The Second Level \\
\hline \multirow{14}{*}{ Index system } & \multirow{4}{*}{ Economic indicator } & The project total investment \\
\hline & & The unit operation cost \\
\hline & & The profit rate of investment \\
\hline & & Land occupation \\
\hline & \multirow{4}{*}{ Technical indicator } & Technology reliability \\
\hline & & Siting requirements \\
\hline & & The processing object requirement \\
\hline & & The operation and management requirement \\
\hline & \multirow{3}{*}{ Environmental indicator } & Environment pollution \\
\hline & & Secondary pollution \\
\hline & & Health and safety evaluation \\
\hline & \multirow{3}{*}{ Social indicator } & Literacy of public \\
\hline & & Public participation level \\
\hline & & Laws and regulations \\
\hline
\end{tabular}

Table 2. Calculation results of judgment matrix A-B layer.

\begin{tabular}{|c|c|c|c|c|c|c|c|}
\hline$A$ & $B 1$ & $B 2$ & $B 3$ & $B 4$ & $\omega_{i}$ & $\lambda_{\max }$ & Consistency check \\
\hline \hline$B 1$ & 1 & 3 & $1 / 5$ & $1 / 2$ & 0.1428 & & $R I=0.90$ \\
\hline$B 2$ & $1 / 3$ & 1 & $1 / 5$ & $1 / 3$ & 0.0747 & 4.1314 & $C I=\left(\lambda_{\max }-n\right) /(n-1)=0.0438$ \\
\hline$B 3$ & 5 & 5 & 1 & 3 & 0.5602 & & $C R=C I / R I=0.0487<0.1$ \\
\hline$B 4$ & 2 & 3 & $1 / 3$ & 1 & 0.2223 & & \\
\hline
\end{tabular}

Table 3. Calculation results of judgment matrix.

\begin{tabular}{|c|c|c|}
\hline Judgment Factors & CR & Weight Coefficient \\
\hline \hline$U=\{B 1, B 2, B 3, B 4\}$ & 0.0487 & $A=(0.1428,0.0747,0.5602,0.2223)$ \\
\hline$U 1=\{C 1, C 2, C 3, C 4\}$ & 0.0039 & $A 1=(0.42,0.23,0.23,0.12)$ \\
\hline$U 2=\{C 5, C 6, C 7, C 8\}$ & 0.0054 & $A 2=(0.27,0.48,0.09,0.16)$ \\
\hline$U 3=\{C 9, C 10, C 11\}$ & 0.0738 & $A 3=(0.1007,0.2255,0.6738)$ \\
\hline$U 4=\{C 12, C 13, C 14\}$ & 0.0553 & $A 4=(0.2524,0.4158,0.3318)$ \\
\hline
\end{tabular}

$$
\begin{gathered}
f_{H}\left(x_{1}\right)=\left\{\begin{array}{c}
1, x \geq a_{1} \\
\left(x-a_{2}\right) /\left(a_{1}-a_{2}\right), a_{2}<x<a_{1} \\
0, x \leq a_{2}
\end{array}\right. \\
f_{G}\left(x_{1}\right)=\left\{\begin{array}{c}
\left(a_{3}-x\right) /\left(a_{3}-a_{4}\right), a_{4}<x<a_{3} \\
1, a_{5} \leq x \leq a_{4} \\
\left(x-a_{6}\right) /\left(a_{5}-a_{6}\right), a_{6}<x<a_{5}
\end{array}\right.
\end{gathered}
$$

$$
\begin{gathered}
f_{M}\left(x_{1}\right)=\left\{\begin{array}{c}
\left(a_{8}-x\right) /\left(a_{7}-a_{8}\right), a_{8}<x<a_{7} \\
1, a_{9} \leq x \leq a_{8} \\
\left(x-a_{10}\right) /\left(a_{9}-a_{10}\right), a_{10} \leq x<a_{9}
\end{array}\right. \\
f_{L}\left(x_{1}\right)=\left\{\begin{array}{c}
\left(a_{11}-x\right) /\left(a_{11}-a_{12}\right), a_{12} \leq x \leq a_{11} \\
1, x<a_{12}
\end{array}\right.
\end{gathered}
$$


Table 4. Comparison table of four disposal methods.

\begin{tabular}{|c|c|c|c|c|}
\hline Comparison of Content & Sanitary Landfill & Hot Composts & Burn & Comprehensive Treatment \\
\hline Unit investment(million/t) & $0.06-0.15$ & $0.05-0.4$ & $0.35-0.65$ & $0.15-0.3$ \\
\hline Operation cost(yuan/t) & $50-80$ & $50-110$ & Around 150 & Around 85 \\
\hline Land occupancy & large & more & less & less \\
\hline Exhaust gas & Produce methane & Acid gas emissions & Produce harmful gases & Heavy metal \\
\hline Waste water & Landfill leachate & Less waste leachate & $\begin{array}{l}\text { The possibility is very } \\
\text { small }\end{array}$ & The possibility is small \\
\hline soil & Leachate pollution & Soil compaction & $\begin{array}{l}\text { Settlement of gaseous } \\
\text { pollutants }\end{array}$ & \\
\hline Applicable condition & All kinds of life garbage & $\begin{array}{c}\text { Perishable goods accounted } \\
\text { for } 40 \%\end{array}$ & $\begin{array}{l}\text { Calorific value greater than } \\
\qquad 4127 \mathrm{KJ} / \mathrm{kg}\end{array}$ & All kinds of life garbage \\
\hline Technology reliability & reliable & More reliable & More reliable & More reliable \\
\hline Safety of operation & $\begin{array}{l}\text { Good, but pay attention to } \\
\text { gas explosion }\end{array}$ & better & better & better \\
\hline Management level & The general requirements & Higher requirements & High requirements & High requirements \\
\hline Literacy of public & The general requirements & Higher requirements & High requirements & High requirements \\
\hline Public participation level & poor & better & good & good \\
\hline Laws and regulations & No reduction & $20 \%$ & More than $80 \%$ & More than $90 \%$ \\
\hline
\end{tabular}

\section{II}

Normal membership function model is described as follows:

$$
\begin{aligned}
& f_{H}(x)=\exp \left[-\left(\frac{x-b_{1}}{\sigma}\right)^{2}\right], f_{G}(x)=\exp \left[-\left(\frac{x-b_{2}}{\sigma}\right)^{2},\right. \\
& f_{M}(x)=\exp \left[-\left(\frac{x-b_{3}}{\sigma}\right)^{2}\right], f_{L}(x)=\exp \left[-\left(\frac{x-b_{4}}{\sigma}\right)^{2}\right]
\end{aligned}
$$

The evaluation set is (excellent, good, medium, poor), and the corresponding score for $(4,3,2,1)$, membership is as follows:

Excellent: $f(x)=\left\{\begin{array}{c}1, x \geq 1.5 \\ (x-1.2) / 0.3,1.2<x<1.5, \\ 0, x \leq 1.2\end{array}\right.$

good: $f(x)=\left\{\begin{array}{c}1, x \geq 1.2 \\ (x-0.9) / 0.3,0.9<x<1.2 \\ 0, x \leq 0.9\end{array}\right.$

$$
\text { medium: } f(x)=\left\{\begin{array}{c}
1, x \geq 0.9 \\
(x-0.6) / 0.3,0.6<x<0.9 \text {, } \\
0, x \leq 0.6
\end{array}\right.
$$

poor: $f(x)=\left\{\begin{array}{c}1, x \geq 0.6 \\ (x-0.3) / 0.3,0.3<x<0.6 \\ 0, x \leq 0.3\end{array}\right.$

\section{FUZZY COMPREHENSIVE EVALUATION PROC-} ESS

We calculate each index membership degree for four classes, two class membership matrix is obtained (Table 5) (including total engineering investment and unit operating costs as low as possible, therefore the calculation results are in reverse order.)

The calculation of the judgement matrix

According to the fuzzy evaluation results and the weight coefficient, as indicated in the table, the first two grade evaluation matrix are calculated from the bottom to the top and compute a level judgment matrix

$$
M_{1 L}=A 1 \circ R_{1 L}=\left(\begin{array}{llll}
0.42 & 0.23 & 0.23 & 0.12
\end{array}\right) \circ
$$

$\left(\begin{array}{cccc}0 & 0.39 & 0 & 0 \\ 1 & 0 & 0 & 0 \\ 0 & 0 & 0 & 0.183 \\ 0.9 & 0.3 & 0.6 & 0.2\end{array}\right)=\left(\begin{array}{llll}0.34 & 0.2 & 0.07 & 0.066\end{array}\right)$

\section{Similarly}

$M_{1 C}=\left(\begin{array}{llll}0.072, & 0.3534, & 0.266, & 0.012\end{array}\right)$

$M_{1 B}=\left(\begin{array}{llll}0.245, & 0.198, & 0.372, & 0.006\end{array}\right)$,

$M_{1 S}=\left(\begin{array}{llll}0.246, & 0.187, & 0.112, & 0.021\end{array}\right)$,

$M_{2 L}=\left(\begin{array}{lll}0.0135, & 0.233,0.287,0.48\end{array}\right)$, 
Table 5. The calculation results of fuzzy comprehensive evaluation.

\begin{tabular}{|c|c|c|c|c|c|}
\hline \multirow{2}{*}{ Evaluation Object } & \multirow{2}{*}{$\begin{array}{c}\text { Membership } \\
\text { Function }\end{array}$} & \multicolumn{4}{|c|}{ The Evaluation Matrix } \\
\hline & & Sanitary Landfill & Composts & Burn & Comprehensive Treatment \\
\hline $\begin{array}{l}\text { The project total } \\
\text { investment }\end{array}$ & I & $(0,0.39,0,0)$ & $(0,0.67,0,0)$ & $(0,0,0.6,0)$ & $(0,0.3,0.1,0)$ \\
\hline The unit operation cost & I & $(1,0,0,0)$ & $(0,0,1,0)$ & $(0,0,0.46,0)$ & $(0,0.21,0,0)$ \\
\hline $\begin{array}{l}\text { The profit rate of } \\
\text { investment }\end{array}$ & II & $(0,0,0,0.183)$ & $(0,0,0,0.001)$ & $(0.7,0.6,0.01,0)$ & $(0.6,0.03,0.3,0.07)$ \\
\hline Land occupation & II & $(0.9,0.3,0.6,0.2)$ & $(0.6,0.6,0.3,0.1)$ & $(0.7,0.5,0.1,0.05)$ & $(0.9,0.05,0.01,0.04)$ \\
\hline Technology reliability & III & $(0.05,0.5,0.5,0)$ & $(0,0.07,0.9,0)$ & $(0,0.07,0.9,0)$ & $(0,0.9,0.1,0)$ \\
\hline Siting requirements & III & $(0,0,0,1)$ & $(0,0,1,0)$ & $(0.3,0.3,0.3,0)$ & $(0.4,0.4,0.2,0)$ \\
\hline $\begin{array}{l}\text { The processing object } \\
\text { requirement }\end{array}$ & III & $(0,0.2,0.8,0)$ & $(0,0,0,1)$ & $(0,0.3,0.7,0)$ & $(0,0.9,0.1,0)$ \\
\hline $\begin{array}{l}\text { The operation and man- } \\
\text { agement requirement }\end{array}$ & III & $(0,0.5,0.5,0)$ & $(0,0.3,0.7,0)$ & $(0,0,1,0)$ & $(0.2,0.4,0.4,0)$ \\
\hline Environment pollution & III & $(0,0,1,0)$ & $(0,0.03,0.97,0)$ & $(0.1,0.4,0.5,0)$ & $(0,0.5,0.5,0)$ \\
\hline Secondary pollution & III & $(0,0,1,0)$ & $(0,0.2,0.8,0)$ & $(0,0.5,0.5,0)$ & $(0,0.6,0.4,0)$ \\
\hline $\begin{array}{l}\text { Health and safety } \\
\text { evaluation }\end{array}$ & III & $(0,0,0,1)$ & $(0,0,1,0)$ & $(0.3,0.3,0.4,0)$ & $(0.1,0.5,0.4,0)$ \\
\hline Literacy of public & III & $(0,0,0,1)$ & $(0,0,1,0)$ & $(0.2,0.4,0.3,0.1)$ & $(0.4,0.3,0.2,0.1)$ \\
\hline $\begin{array}{c}\text { Public participation } \\
\text { level }\end{array}$ & III & $(0,0,0,1)$ & $(0,0,1,0)$ & $(0.2,0.4,0.4,0)$ & $(0.4,0.3,0.3,0)$ \\
\hline Laws and regulations & III & $(0.2,0.4,0.4,0)$ & $(0,0.2,0.8,0)$ & $(0,0,1,0)$ & $(0,0.8,0.2,0)$ \\
\hline
\end{tabular}

$M_{2 C}=\left(\begin{array}{llll}0, & 0.067, & 0.835, & 0.09\end{array}\right)$,

$M_{2 B}=\left(\begin{array}{llll}0.144, & 0.19, & 0.610\end{array}\right)$,

$M_{2 S}=\left(\begin{array}{lll}0.224, & 0.580,0.196,0\end{array}\right)$,

$M_{3 L}=\left(\begin{array}{llll}0, & 0, & 0.3262 & 0.6738\end{array}\right)$,

$M_{3 C}=\left(\begin{array}{lll}0,0.048, & 0.9519,0\end{array}\right)$,

$M_{3 B}=\left(\begin{array}{lll}0.2122, & 0.3653,0.2700,0\end{array}\right)$,

$M_{3 S}=\left(\begin{array}{llll}0.0674, & 0.4266 & 0.4100,0\end{array}\right)$,

$M_{4 L}=\left(\begin{array}{llll}0.066, & 0.133, & 0.133,0.668\end{array}\right)$

$M_{4 C}=\left(\begin{array}{lll}0,0.066,0.266,0 & 0\end{array}\right)$

$M_{4 B}=\left(\begin{array}{llll}0.134, & 0.267,0.574,0.025\end{array}\right)$,

$M_{4 S}=\left(\begin{array}{llll}0.267, & 0.466, & 0.441, & 0.025\end{array}\right)$.

Then the two level evaluation matrix is formed as follows:

$$
M_{L}=\left(\begin{array}{l}
M_{1 L} \\
M_{2 L} \\
M_{3 L} \\
M_{4 L}
\end{array}\right)=\left(\begin{array}{cccc}
0.34 & 0.2 & 0.07 & 0.066 \\
0.0135 & 0.233 & 0.287 & 0.480 \\
0 & 0 & 0.3262 & 0.6738 \\
0.066 & 0.133 & 0.133 & 0.668
\end{array}\right)
$$

Similarly,

$$
M_{C}=\left(\begin{array}{l}
M_{1 C} \\
M_{2 C} \\
M_{3 C} \\
M_{4 C}
\end{array}\right), M_{B}=\left(\begin{array}{l}
M_{1 B} \\
M_{2 B} \\
M_{3 B} \\
M_{4 B}
\end{array}\right), M_{S}=\left(\begin{array}{l}
M_{1 S} \\
M_{2 S} \\
M_{3 S} \\
M_{4 S}
\end{array}\right)
$$

Then according to the weight of index economy, technology, environment, and society

$$
A=\left(\begin{array}{llll}
0.1428, & 0.0747, & 0.5602, & 0.2223
\end{array}\right)
$$

Overall evaluation results can be obtained as $G=A \circ M$

$$
G_{L}=\left(\begin{array}{llll}
0.064, & 0.076, & 0.244 & 0.571
\end{array}\right),
$$

$G_{C}=\left(\begin{array}{llll}0.010, & 0.097, & 0.693, & 0.008\end{array}\right)$

$G_{B}=\left(\begin{array}{llll}0.194, & 0.306, & 0.378, & 0.006\end{array}\right)$

$G_{S}=\left(\begin{array}{llll}0.149, & 0.413, & 0.389, & 0.008\end{array}\right)$

This still does not depict the advantages of four methods, take on the four levels of quantitative score

$D=(4, \quad 3, \quad 2,1)^{T}$

$T=G \circ D$, we can get

$T_{L}=G_{L} \circ D=1.543, T_{C}=G_{C} \circ D=1.725$, 


$$
T_{B}=G_{B} \circ D=2.456, T_{S}=G_{S} \circ D=2.621
$$

From the above results, we can see that, the integrated treatment has the highest scores and is most suitable comprehensive treatment method for Shunyi garbage disposal plant construction.

\section{CONCLUSION}

This paper takes waste treatment plant as research object and arrives at the conclusion that the most suitable treatment method for the plant is comprehensive waste disposal method. Comprehensive waste disposal method has all the advantages of landfill, compost, incineration, thus has become general trend of waste disposal worldwide.

\section{CONFLICT OF INTEREST}

The authors confirm that this article content has no conflict of interest.

\section{ACKNOWLEDGEMENTS}

The paper is financially supported by key Science and Technology Fund of Agricultural University of Hebei 2014
(ZD201406), Science and Technology Project of Hebei Province (12453804,13454707D).

\section{REFERENCES}

[1] Keith Knox, Knox Associates. Sustainable landfill in the UK[R]. vol. 202, 2000

[2] L. F, Diaz, G. M. Savage, and C. G. Golueke, "Resource Recovery from Municipal Solid Waste Primary Processing," CRC Press, Inc, pp. 30-32, 1982.

[3] J. Hu, "A study on Evaluation of Municipal Solid Waste Treatment Model," Huazhong University of Science and Technology, Wuhan 430074, P.R.China, 2008.

[4] N.B. Chang, G. Parvathinathanb, and J. B. Breeden, "Combining GIS with fuzzy multicriteria decision-making for landfill sitting in a fast-growing urban region," Journal of Environmental Management, vol. 87, pp. 139-153, 2008.

[5] M. Crest, D. Blanc, P. Moszkowicz, and C. Dujet, "Experimental percolation under intermittent conditions: Influence on pollutants emission from waste," Journal of Hazardous Materials, vol. 139, pp. 523-528, 2007.

[6] Chinese Environmental Protection Industry Association of Municipal Solid Waste Disposal Committee, "Development Report on Municipal Solid Waste Disposal Industry in 2008 in China," Environmental Protection Industry In China, vol. 6, pp. 17-23, 2009.

[7] G. Hu, X. Ma, and Y. Liu, "Comprehensive Evaluation System for Residential Refuse Treatment Model of Small Towns in Three Gorges Area," Environmental Science and Management, In China, vol. 37, pp.180-185, 2012

Received: May 26, 2015

Revised: July 14, 2015

Accepted: August 10,2015

(C) Zhang et al.; Licensee Bentham Open.

This is an open access article licensed under the terms of the (https://creativecommons.org/licenses/by/4.0/legalcode), which permits unrestricted, noncommercial use, distribution and reproduction in any medium, provided the work is properly cited. 